\title{
Supporting Collaborative Sensemaking in Map-Based Emergency Management and Planning
}

\author{
Anna Wu \\ College of Information Science \& Technology \\ Pennsylvania State University \\ University Park, PA, 16802 \\ 814-863-6822 \\ annawu@psu.edu
}

\author{
Advisor: Xiaolong Zhang \\ College of Information Science \& Technology \\ Pennsylvania State University \\ University Park, PA, 16802 \\ 814-863-9462 \\ Izhang@ist.psu.edu
}

\begin{abstract}
Emergency management and planning often involves multiple domain experts with diverse knowledge backgrounds and responsibilities. Current practices in emergency management and planning have not leveraged the state-of-art technologies in information sharing, synthesis, and analysis. The proposed research will investigate the process of collaborative sensemaking in emergency planning and implement a mapbased online system to support this process.
\end{abstract}

\section{Categories and Subject Descriptors}

H.5.3. [Information interfaces and presentation (e.g., HCI)] Group and Organization Interfaces: Computer-supported cooperative work

General Terms: Measurement, Design, Experimentation Keywords: Collaborative sensemaking, Geo-collaboration, supportive system

\section{INTRODUCTION OF THE PROBLEM}

In emergency management planning, collaborative decision usually involves experts with different knowledge backgrounds. To better understand task situations and coordinate work, team members need to individually analyze massive data from various sources by leveraging their domain expertise. The success of collaborative sensemaking largely relies on the effectiveness of sharing relevant information among domain experts and integrating diverse inputs into a coherent plan $[1,2]$. However, factors like domain barrier, temporal disjunction, geographical dispersion, and media diversity make collaborative sensemaking in emergency management a big challenge. Moreover, emergency planning usually involves geospatial information, which is difficult to communicate verbally.

To augment human intelligence and maximize the use of human knowledge, computational tools can be helpful because they offer large storage capacity, high retrieval efficiency, fast processing speed, and broadband communication. My research question is how to support collaborative sensemaking in emergency management with visualization tools.

\section{PREVIOUS WORK}

Collaborative sensemaking is important in emergency

Copyright is held by the author/owner(s). GROUP'09, May 10-13, 2009, Sanibel Island, Florida, USA. ACM 978-1-60558-500-0/09/05. management and planning, where people encounter large amount of information and need to make sense of 'what is going on'. In collaborative sensemaking, information artifacts are often first collected, assessed, and filtered by individuals, and then those artifacts that are relevant and important to the group are integrated and discussed by the whole group. Moving from individual sensemaking to collaborative sensemaking raises some challenges.

Current collaboration systems (e.g. email, video-conference, VoIP, etc.) usually do not support collaborative sensemaking directly. Though these tools can support social awareness among distributed group members, most of these systems focus on widening the communicative band, but conveying information does not necessarily result in the mutual understanding of the meaning, which is especially true when the inference needs domain expertise. In highly specialized emergency situations, such as emergency room in the hospital, to achieve collaborative sensemaking, people make use of whatever possible artifacts to increase articulation [3].

Societal planning tasks are inherently wicked problems which are ill-defined and without clear stopping rule [4]. Visual analytic is a key technique to get insight of this kind of wicked problems [5]. Dynamic nature and complex interdependent constraints in emergency planning determine that collaborative problem-solving will only work if planners succeed in coordinating their problem representations, their inference processes, and the final results they gain. Visualizing the analytic and reasoning processes could benefit the collaborators to have coherent representations, focused awareness, and consistent, accurate evaluation of the solution [6]. Interactive visualization, especially, allows users editing and exploring their own interested information with various perspectives based on individual collaborators' responsibilities. For the purpose of collaboration, the visualized data can be immediate, intermediate, or final results in individual decision process, which is exchangeable between collaborators.

Emergency management organizes resources and responsibility across different locations to deal with emergency situations. Most of the emergency management planning, if not all, involves geospatial information that can be located on maps. Thus, we assert that emergency management and planning is also a collaborative spatial decision making problem. Research suggests that Geography Information Systems (GIS) can help decision making in emergency management [e.g. 7, 8]. However, these GIS related research focuses more on generating geographic information, rather than supporting the integration of spatial information to augment collaborative sensemaking and group decision making. Also, the accessibility and usability of 
these systems are usually problematic for distributed group members in emergency management.

\section{PROPOSED APPROACH}

Thus, my proposed approach is to incorporate map services with visualization tools to help collaborators understanding complex information in emergency management and planning. The research will combine two components: system development, and fieldwork. The goal of system development is to design and implement a web-based system to support collaborative sensemaking and decision-making with visualization tools. The system will integrate online map services (e.g., Google Maps) and provide users with a set of visual analytic tools. The purposes of fieldwork are to improve our understanding of the practices in emergency management and planning, to collect user feedback on our system, and to evaluate our system. We will observe the usage behavior and carry out focus-group interview to collect behavior information using this system.

These two components are in an iterative process, in which user requirements and needs are consistently collected through fieldwork and then integrated into new designs, see Figure 1Error! Reference source not found. This iterative process may be repeated until we have a good understanding of behavior model and the system is ready to be deployed. Based on the system and empirical data we collect through fieldwork, we will eventually develop a theoretical model of collaborative sensemaking in emergency situation and develop design guidelines for similar supportive systems.

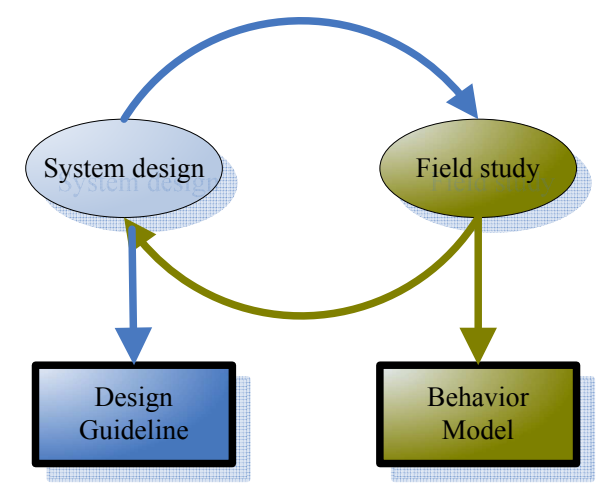

Figure 1 Proposed Approach

Currently, based on our previous field research, an online prototype has been implemented (See an online demo at http://zhang.ist.psu.edu/Research/Demo/Googlemap/file165-

1.avi). Preliminary lab evaluation has been conducted. The prototype has been demonstrated to emergency managers from the Department of Homeland Security and local government agencies at central Pennsylvania. In additional to positive feedback, these emergency management professional also provide constructive suggestions on system improvement to accommodate fieldwork.

\section{EXPECTED RESULTS AND QUESTOINS}

The expected results of this research are two-folded. First, I will develop a behavioral model of collaborative sensemaking. This model will identify key factors and processes in collaborative sensemaking. This model will inform designs of systems to support similar sensemaking activities in other situations, such as intelligence analysis. Second, I expect the web-based collaborative sensemaking system derived from my research will transform the practices of emergency management and planning professionals and improve the efficiency and effectiveness of their work on analyzing and synthesizing diverse information.

Several issues remain at this stage. The first issue is related to the use of common representations in collaborative sensemaking. Common representations server as communicational media shared by all the group members, and are critical to collaboration tasks. Even we have identified the general constructs in emergency managements, how to represent these constructs so that all team members can create, understand, and manipulate them without large cognitive workload is unclear. The second challenge is how to generalize visualization designs. Visualization designs currently seen in our prototype rely on structured data with well-defined attributes, which can be easily processed (e.g., clustering and aggregation). However, in emergency management and planning, data may come without structures or not wellstructured. Then, discovering intrinsic organization schemes of data, which is required by visualization, becomes a challenge. Third, integrating maps with other visualization tools is a new area with many unanswered questions. A map not only provides geographic information but is a visual representation itself. By adding other visual components, such as aggregation layers, to maps, we face a question about to what extent the integration can support collaborative problem solving without increasing cognitive burdens.

\section{REFERENCES}

[1] Schafer, W. A., et al. Supporting Community Emergency Management Planning through a Geocollaboration Software Architecture. Computer Supported Cooperative Work (CSCW), 16, 4 (2007), 501-537.

[2] Convertino, G., et al. Designing Group Annotations and Process Visualizations for Role-Based Collaboration. SBP'08 (2008), 1-10.

[3] Paul, S. A. and Reddy, M. C. Information and communication tools as aids to collaborative sensemaking,(2008).

[4] Rittel, H. and Webber, M. Dilemmas in a General Theory of Planning. Policy Sciences, 4,(1973), 155-169.

[5] Kirschner, P. A. Visualizing Argumentation: Software Tools for Collaborative and Educational Sense-Making. Springer, 2003.

[6] Alpay, L., et al. Accidentology: An example of problem solving by multiple agents with multiple representations. Elsevier, Oxford, UK, 1998.

[7] Rauschert, I., et al. Designing a human-centered, multimodal GIS interface to support emergency management, ACM New York, NY, USA. (2002), 119124.

[8] Zerger, A. and Smith, D. I. Impediments to using GIS for real-time disaster decision support. Computers, Environment and Urban Systems, 27, 2 (2003), 123-141. 